\title{
Effect of a quartic anisotropy energy on the "spiral magnetic" coexistence state of superconductivity and ferromagnetism
}

\author{
Gregory H. Rose and Chia-Ren Hu \\ Center for Theoretical Physics, Department of Physics, Texas A\&M University, College Station, Texas 77843 \\ (Received 26 June 1987)
}

\begin{abstract}
The effect of three types of "quartic" anisotropy energy (i.e., in the $M^{4}$ term of the magnetic Ginzburg-Landau free energy) on the polarization of the "spiral magnetic" state of Blount and Varma is studied near the onset temperature. For a quartic anisotropy with uniaxial symmetry, we find continuous polarization transitions from circular to elliptical and then to linear as the strength of a uni-easy-axis anisotropy is increased. (No transition is found for the uni-hard-axis case.) If the quartic anisotropy has cubic symmetry, we find a discontinuous transition directly between circular and linear, without going through an elliptic stage, when the sign of the anisotropy energy is to favor the cubic axes. (The polarization stays circular at all strengths of the anisotropy energy if the sign of the latter is to favor the body diagonals.) Finally, we model the anisotropy in primitive tetragonal $\mathrm{ErRh}_{4} \mathrm{~B}_{4}$ with a quadratic anisotropy giving a hard $c$ axis, plus a quartic anisotropy in the basal plane with a square symmetry. A first-order polarization transition directly between circular and linear is also obtained for this case, when the quartic anisotropy favors the principal axes in the basal plane. This last case studied provides a plausible explanation for the linear polarization observed in the coexistence state of $\mathrm{ErRh}_{4} \mathrm{~B}_{4}$.
\end{abstract}

\section{INTRODUCTION AND SUMMARY}

Using an isotropic Ginzburg-Landau theory to investigate the interaction of superconductivity and ferromagnetism via the magnetic field $\mathbf{B}$, Blount and Var$\mathrm{ma}^{1}$ predicted a new "spiral magnetic" (SM) state which features a coexistence of superconductivity and a spiraling spontaneous magnetization of a wave length much longer than the lattice spacing. Assuming that the bare superconducting transition temperature $T_{c}$ is substantially higher than the bare ferromagnetic transition temperature $T_{m 0}$, they found the SM state to occur in a temperature range $T_{c 2}<T<T_{s}$, where $T_{s}$ is only slightly below $T_{m 0}$, and below $T_{c 2}$ a normal ferromagnetic state becomes the most stable state. According to this theory, either temperature region may or may not exist depending on the values of several material parameters that have been introduced in the theory. The theory was presumed to apply to such ternary "reentrant" superconductors as $\mathrm{ErRh}_{4} \mathrm{~B}_{4}$ (the primitive tetragonal kind) and $\mathrm{HoMo}_{6} \mathrm{~S}_{8}$, etc., and indeed in subsequent small-angle neutron scattering experiments performed on powdered samples of these materials, ${ }^{2,3}$ a peak was observed at a small but finite wave number $q$ which appears to be consistent with the theory. Further experiments performed on a single crystal of $\mathrm{ErRh}_{4} \mathrm{~B}_{4},{ }^{4,5}$ however, revealed that this material has a tetragonal symmetry, is strongly anisotropic, and its oscillatory spontaneous magnetization is confined to either of the two $a$ axes in the tetragonal basal plane, and therefore is linearly polarized. As for $\mathrm{HoMo}_{6} \mathrm{~S}_{8}$, it is known ${ }^{6}$ that this Chevrel-phase compound has rhombohedral symmetry which may be thought of as a slightly distorted cubic symmetry with the distortion being a slight elongation along one of the cubic diagonals. Neutron scattering and magnetic measurements have determined that the trigonal axis is the easy axis for magnetization, and $q \|[110] .^{7}$ The coexistence state in this material is therefore also linearly polarized.

In order to accommodate these observed linear polarizations into the Blount-Varma theory, Greenside et al. investigated in Ref. 8 the effect of a "quadratic" anisotropy of the form $\frac{1}{2}\left[\alpha_{\|} M_{z}^{2}+\alpha_{1}\left(M_{x}^{2}+M_{y}^{2}\right)\right]$ on the SM phase. This form is to replace the isotropic term $\frac{1}{2} \alpha_{m}|\mathbf{M}|^{2}$ in the original isotropic magnetic-GinzburgLandau free energy of Blount and Varma. For $\alpha_{\|}=\alpha_{\| 0}\left(T / T_{m 0}^{\|}-1\right), \quad \alpha_{\perp}=\alpha_{10}\left(T / T_{m 0}^{1}-1\right)$, with $T_{m 0}^{\|}$ $\neq T_{m 0}^{1}$, this form represents an anisotropic bare Curie temperature. For $T_{m 0}^{\|}=T_{m 0}^{1}=T_{m 0}$ but $\alpha_{\| 0} \neq \alpha_{10}$, this form represents an anisotropic Curie constant for $T>T_{m 0}$. Without the need to distinguish between the two cases, Greenside et al. found that the circularly polarized (CP) SM state changed to an elliptically polarized (EP) state and then to a linearly polarized (LP) state as $\alpha_{\|} / \alpha_{\perp}$ was increased from unity to eventually exceed $\approx 1.8$, where the LP state sets in. Clearly their theory applies to the regime $T<T_{m 0}^{1} \leq T_{m 0}^{\|}$, whereas for $T_{m 0}^{\perp}<T<T_{m 0}^{\|}$the polarization must be trivially linear since only the $z$ component of $\mathbf{M}$ can be nonzero in this regime. For cubic materials, $\alpha_{\|}$and $\alpha_{1}$ are necessarily equal. The important anisotropy to consider is then a "quartic" anisotropy of the form $\frac{1}{4} \beta^{\prime}\left(M_{x}^{4}+M_{y}^{4}+M_{z}^{4}\right)$, to be added to the isotropic term $\frac{1}{4} \beta_{m} \mathbf{M}^{4}$. [The sum of these two terms may also be written as

$$
\frac{1}{4}\left(\beta_{m}+\beta^{\prime}\right) \mathbf{M}^{4}-\frac{1}{2} \beta^{\prime}\left(M_{x}^{2} M_{y}^{2}+M_{x}^{2} M_{z}^{2}+M_{y}^{2} M_{z}^{2}\right),
$$

and is therefore equivalent to the anisotropy considered 
by Kuper et $\left.a l .{ }^{9}\right]$ This raises the curious question as to whether such a quartic anisotropy is also capable of inducing a polarization transition of the type found by Greenside et al. This is the initial motivation of this study, but we have extended our study to include the effect of a quartic anisotropy with a uniaxial symmetry in order to reveal a different type of quartic anisotropy effect on the polarization transition, and we have also modeled the anisotropy of the actual primitivetetragonal $\mathrm{ErRh}_{4} \mathrm{~B}_{4}$ within the Ginzburg-Landau approach used here, and then study its effect on the polarization transition of this type of material as the strength of the anisotropy energy is increased. The purpose of this last part of the study is to find a plausible explanation why linear polarization is actually observed in the coexistence state in $\mathrm{ErRh}_{4} \mathrm{~B}_{4}$, in spite of the fact that $T_{m 0}$ and the magnetic susceptibility are both isotropic in the tetragonal easy-basal plane of this material. Clearly this question is not answered by the anisotropy effect studied by Greenside et al., since they have only considered the effect of an easy axis in the quadratic term of the magnetic free energy. It is also clear that if the easy axis is changed to a hard axis in their model without the addition of any other anisotropy energy, the polarization of the spiral magnetic state will remain circular (though with $q$ now confined to the hard axis), no matter what the strength of this quadratic anisotropy. Thus, in order to explain the linear polarization observed in this material we have modeled the anisotropy of this material by adding to the quadratic hard-axis anisotropy described above by a quartic anisotropy of square symmetry in the tetragonal basal plane. Indeed within this model we find a direct first-order polarization transition from circular to linear, as the strength of this quartic anisotropy is increased, if its sign is to cause the magnetization to favor either of the two $a$ axes. We thus have obtained a plausible explanation of the linear polarization observed in the coexistence state of $\mathrm{ErRh}_{4} \mathrm{~B}_{4}$, although we do not claim that the model used here has been established as the correct model for the anisotropy energy of this material (i.e., more complex models consistent with the crystal symmetry of this material can certainly be constructed). On the other hand, Chevrel-phase materials such as $\mathrm{HoMo}_{6} \mathrm{~S}_{8}$ have a single strong easy axis along the trigonal axis. It is clear then that the anisotropy effect studied by Greenside $e t$ al. can already explain the linear polarization observed in this class of materials, and no special consideration in this study is necessary for these materials. (Actually it is the trivial case $T_{m 0}^{1}<T<T_{m 0}^{\|}$which applies, so even the theory of Greenside et al. is also not necessary for these materials).

Thus, in this work, we have studied the effects of three types of quartic anisotropy: (i) The uniaxial quartic anisotropy (case 1) is of the form $\frac{1}{4} \beta^{\prime} M_{z}^{4}$ which is added to $\frac{1}{4} \beta_{m} \mathbf{M}^{4}$. It gives continuous polarization transitions of the form $\mathrm{CP} \rightarrow \mathrm{EP} \rightarrow \mathrm{LP}$ as $\beta^{\prime} / \beta_{m}$ is varied from 0 down to -1 (the stability limit). (On the other hand, no transition is found for $\beta^{\prime}>0$.) (ii) The quartic anisotropy with cubic symmetry (case 2) is of the form $\left(\beta^{\prime} /\right.$ 4) $\left(M_{x}^{4}+M_{y}^{4}+M_{z}^{4}\right)$, again to be added to $\frac{1}{4} \beta_{m} \mathbf{M}^{4}$. We find a polarization transition of the form $\mathbf{C P} \rightarrow \mathrm{LP}$ (without going through an EP stage), for which the first derivative of the free energy with respect to $\beta^{\prime}$ is discontinuous, as $\beta^{\prime} / \beta_{m}$ is again lowered from 0 to -1 . (Again, no transition is found for $\beta^{\prime}>0$.) Finally, (iii) we study a quartic anisotropy with the symmetry of primitive tetragonal $\mathrm{ErRh}_{4} \mathrm{~B}_{4}$ (case 3), which is of the form $\left(\beta^{\prime} / 4\right)\left(M_{x}^{4}+M_{y}^{4}\right)$. This is to be considered together with the assumption that the $z$ axis is hard (i.e., the onset temperature for ferromagnetism in the $z$ direction is much lower than those for ferromagnetism in the $x$ and $y$ directions). This case gives a phase transition very similar to case 2 of the form $\mathrm{CP} \rightarrow \mathrm{LP}$, again without going through an EP stage. (For $\beta^{\prime}<0$, otherwise it is always CP.) Thus in terms of the standard nomenclature we may term the transitions for cases 2 and 3 first-order transitions, whereas the transitions for the uniaxial quartic anisotropy, as well as for the quadratic anisotropy analyzed by Greenside et al., may be termed secondorder transitions. In all three cases, the critical value of $\beta^{\prime} / \beta_{m}$ for entering linear polarization was found to be $-\frac{2}{3}$, but for case 1 there is another critical value at 0 , where EP degenerates into CP. Strictly speaking, this is not a polarization transition since the $C P$ phase exists for only a single value of $\beta^{\prime}$ rather than a finite range. $(0$ is also a critical value for cases 1 and 2 in another sense: The directions of $\mathbf{q}$ and $\mathbf{M}$ change discontinuously without changing the circular polarization as $\beta^{\prime}$ changes sign from $0_{-}$to $0_{+}$. ) The favored directions for $q$ and $\mathbf{M}$ at each value of $\beta^{\prime} / \beta_{m}$ are given in the subsequent sections and summarized in a table.

Our method of study is based on a near-onset analysis developed previously by one of us, ${ }^{10}$ which is valid only for $T_{s}-T \ll T_{s}$. This restriction greatly reduces the amount of numerical work needed, and permits us to obtain a complete analysis of the polarization transition involved, and the preferred directions for the magnetization $\mathbf{M}$ and the wave vector $q$ for the whole range of the anisotropy parameter $-1 \leq \beta^{\prime} / \beta_{m}<\infty$. In Ref. 10 this analysis was used to study the LP state in the limit of very strong anisotropy to render this state more stable than either the (CP) SM state, or the EP state. Here the analysis is generalized to allow for circular or elliptical polarization. We note that if the condition $T_{s}-T \ll T_{s}$ were not assumed, the oscillatory magnetization of the LP and EP states would be strongly anharmonic, and must be obtained by numerically solving a coupled set of nonlinear differential equations with periodic boundary conditions. This is possible but very time consuming, especially for case 2 , since we wish to determine the directions of $\mathbf{q}$ and $\mathbf{M}$ by minimizing the total free energy. (This problem does not exist in the work of Ref. 8, which is why it can treat lower temperatures.) Thus we have avoided this by confining this study to the vicinity of the onset temperature. We feel that sacrificing a wider validity range of temperature for a much simpler analysis is worthwhile, since there is no experimental indication that a polarization transition can be induced by a mere change of temperature, and for $\mathrm{ErRh}_{4} \mathrm{~B}_{4}$ and $\mathrm{HoMo}_{6} \mathrm{~S}_{8}$ the coexistence state only occurs in a very narrow temperature range anyway. In Ref. 10 an investiga- 
tion was also made of the possible occurence of a more complex doubly periodic structure with negative result. We have not done the same here mainly because we do not yet know how to do that analysis correctly for a general polarization (i.e., we do not yet know how to perceive the most general polarization for a doubly periodic state). Thus, in this study we have confined the magnetization to have a single wave vector $q$, and only analyze the equilibrium polarization of this singly periodic state at each strength of the anisotropy energy.

Before we close this section, we would like to mention some additional experimental facts that are relevant to this study. We have already mentioned that $\mathrm{HoMo}_{6} \mathrm{~S}_{8}$ has a rhombohedral symmetry, with $\mathbf{M}$ being LP along the trigonal (111) axis, and $q \|[1 \overline{1} 0]$. It has been stressed in the literature that even a $70-\mathrm{kG}$ applied field can not induce a full moment perpendicular to the easy axis, ${ }^{11}$ so this material appears to be quite uniaxial, even though a quartic anisotropy term may still be important in quantitatively determining the free energy of this material.

Another Chevrel-phase compound, $\mathrm{HoMo}_{6} \mathrm{Se}_{8}$, has very similar properties. It also has a nearly cubic symmetry with, in fact, a slightly stronger distortion, ${ }^{6}$ and it also exhibits an oscillatory magnetic phase which coexists with superconductivity but with no reentrant behavior. ${ }^{12}$ However, there is some evidence that this material may be less uniaxial. For example, the small-angle neutron scattering intensity for this material peaks at a finite wave vector $q$ with a temperature dependence which can actually be understood in terms of the theory of the CP SM phase, ${ }^{12}$ and yet according to Greenside et al. ${ }^{8}$ the temperature dependence of $\mathbf{q}$ for the LP phase should be substantially stronger than for the CP SM phase. Thus it may very well be true that the anisotropy for this material is more of the cubic symmetry type than uniaxial, although the ultimate test lies in further experiments.

The reentrant superconductor $\mathrm{ErRh}_{4} \mathrm{~B}_{4}$ is, on the other hand, tetragonal with the $c$-axis basis vector nearly 1.4 times longer than the $a$-axes basis vectors. ${ }^{13}$ In Ref. 4 , it was shown that the $c$-axis magnetization is linear in the driving magnetic field even at $0.43 \mathrm{~K}$. This fact establishes that $T_{m 0}^{(z)}$ is much lower than $T_{m 0}^{(x)}$ and $T_{m 0}^{(y)}$ for this material. In Ref. 5, it was shown that the magnetization is linearly polarized in either of the two equivalent $a$ axes in the tetragonal basal plane, and that the $\mathbf{q}$ vector of the coexistence state observed is essen- tially at $45^{\circ}$ with the $c$-axis in either of the two equivalent ac planes. In this work we try to understand the former but not the latter, at least in the sense of explaining it with a physically reasonable model. Since our model is by no means established or unique, we urge the experimentalists to perform the appropriate experiments in order to see whether the basal plane magnetic properties of this material can be understood in terms of such an anisotropy energy model and thereby check the validity of this work concerning this material.

\section{GINZBURG-LANDAU FREE-ENERGY FUNCTIONAL WITH QUARTIC ANISOTROPY}

We consider the effect of adding to the isotropic Ginzburg-Landau free-energy functional for ferromagnetic superconductors [i.e., Eq. (1) of Ref. 10] the following quartic anisotropy energy terms:

$$
\begin{aligned}
& \frac{\beta^{\prime}}{4} M_{z}^{4} \quad(\text { case } 1), \\
& \frac{\beta^{\prime}}{4}\left(M_{x}^{4}+M_{y}^{4}+M_{z}^{4}\right) \quad(\text { case } 2), \\
& \frac{\beta^{\prime}}{4}\left(M_{x}^{4}+M_{y}^{4}\right) \quad(\text { case } 3),
\end{aligned}
$$

where for case three we also change the quadratic term $\frac{1}{2} \alpha \mathbf{M}^{2}$ to $\frac{1}{2}\left[\alpha_{\|} \mathbf{M}_{z}^{2}+\alpha_{\perp}\left(\mathbf{M}_{x}^{2}+\mathbf{M}_{x}^{2}\right)\right]$, with $\alpha_{\|}$finite and also $>0$ and $\alpha_{1} \rightarrow 0$ as $T \rightarrow T_{m 0}$ ( $\left.\equiv T_{m 0}^{\perp}\right)$. Since our study is confined to the vicinity of the onset temperature, the effect of this change is to simply let $\mathbf{M}^{2} \equiv M_{x}^{2}+M_{y}^{2}$, and continue to use the isotropic form $\frac{1}{2} \alpha \mathbf{M}^{2}$, where $\alpha$ now clearly stands for $\alpha_{1}$. We assume that $\beta_{m} \neq 0$ and neglect the $|M|^{6}$ term in this work as a simple model for this effect. Since the magnetization is to be in a general direction, we can write

$$
M_{i}=\frac{M_{i}}{M_{\infty}}(i=x, y, z) .
$$

It is trivial to see that (in the notations of Ref. 10)

$$
\frac{\left(\beta^{\prime} / 4\right) M_{\infty}^{4}}{F_{s 0} \lambda^{3}}=\frac{\beta^{\prime}}{\beta_{m}}\left(\frac{\mu}{v}\right)^{2} .
$$

So the magnetic part of the reduced free-energy functional now becomes [compare with Eq. (6) of Ref. 10]

$$
\Delta \overline{\mathscr{F}}_{\text {magnetic }}=(\mu / v)^{2} \int d^{3} x\left(-2 \mathcal{M}^{2}+\mathcal{M}^{4}+2 \zeta^{-2}|\nabla \boldsymbol{M}|^{2}+\left(\beta^{\prime} / \beta_{m}\right) \times\left\{\begin{array}{l}
M_{z}^{4} \text { for case } 1 \\
\left(\mathcal{M}_{x}^{4}+\mathcal{M}_{y}^{4}+\mathcal{M}_{z}^{4}\right) \text { for case } 2 \\
\left(\mathcal{M}_{x}^{4}+\mathcal{M}_{y}^{4}\right) \text { for case } 3
\end{array}\right\}\right),
$$

where $M^{2} \equiv M_{x}^{2}+M_{y}^{2}+M_{z}^{2}$ for cases 1 and 2 , and $M^{2} \equiv M_{x}^{2}+M_{y}^{2}$ for case 3 .

\section{RIGHT-AT-ONSET ANALYSIS}

The addition of a quartic anisotropy energy term and the extension to a general direction of magnetization in the Ginzburg-Landau free-energy functional will actually lead to the same conclusions for the right-at-onset analysis as in Ref. 10 as we will show below. After varying $\Delta \overline{\mathscr{F}}$ with respect to $f, \mathscr{B}$, and $\boldsymbol{M}$ (with notations of Ref. 10), the additional anisotropy term only gives rise to some cubic terms in $\mathcal{M}_{i}$ and therefore does not appear in the linearized equations. However, now that $\mathcal{M}$ and 
$\mathscr{B}$ are in general directions, we obtain the following equation in place of Eq. (14) of Ref. 10: with

$$
-\mathbf{q}(\mathbf{q} \cdot \sigma \boldsymbol{M})+q^{2} \sigma \mathcal{M}+(\sigma+1) \boldsymbol{M}=0,
$$

$$
\sigma \equiv\left[(v \zeta)^{-2}+\bar{\eta}_{2}\right] q^{2}+\bar{\eta}_{1}-v^{-2}
$$

After taking a scalar product of both sides with $\mathbf{q}$, Eq. (5) simply becomes

$$
(\sigma+1)(\mathbf{q} \cdot \boldsymbol{M})=0,
$$

which implies that either $\sigma+1=0$ or $M \perp$ q. We can now distinguish between two types of solutions:

\section{A. Longitudinal: $(\boldsymbol{M} \| \mathbf{q})$}

We have from Eq. (7)

$$
\sigma+1=0,
$$

and therefore

$$
v^{-2}=\left[(v \zeta)^{-2}+\bar{\eta}_{2}\right] q^{2}+\bar{\eta}_{1}+1 .
$$

This is trivially minimized by the choice of $q^{2}=0$ with the resulting onset temperature too low to be of interest here. [Remember that $v^{-2}=v_{0}^{-2}\left(1-T / T_{m 0}\right)$ and $T=T_{s}$ here.]

\section{B. Transverse: $(\boldsymbol{M} \perp \mathbf{q})$}

Equation (5) then becomes

$$
\left(q^{2}+1\right) \sigma+1=0 \text {, }
$$

which implies

$$
v^{-2}=\left[(v \zeta)^{-2}+\bar{\eta}_{2}\right] q^{2}+\bar{\eta}_{1}+\frac{1}{\left(q^{2}+1\right)} .
$$

This is identical to Eq. (15) of Ref. 10 so the minimization of it with respect to $q$ gives the same onset temperature $T_{s}$ as before:

$1-\frac{T_{s}}{T_{m 0}}=v_{0}^{2}\left\{\bar{\eta}_{1}+2\left[(v \zeta)^{-2}+\bar{\eta}_{2}\right]^{1 / 2}-\left[(v \zeta)^{-2}+\bar{\eta}_{2}\right]\right\}$.

Note that no third mixed situation can occur since as long as $\mathbf{q} \cdot \boldsymbol{M} \neq 0$ then $\sigma+1=0$ and Eq. (5) implies $\boldsymbol{M} \| \mathbf{q}$.

\section{JUST-BELOW-ONSET ANALYSIS}

We now consider temperature $T$ slightly away from $T_{s}$, with $\left|T-T_{s}\right| \ll T_{s}$. The only significant change in the non-linear variational equations appears in the variation of $\Delta \overline{\mathscr{F}}$ with respect to $\mathcal{M}$. After putting $\mathcal{M}=\mathcal{M}_{0}+\delta \mathcal{M}$, neglecting terms to higher than first order in $\delta M$ and simplifying it by using the equation for $M_{0}$ which is defined in Ref. 10, we find that for case 2, Eq. (20c) of Ref. 10 is changed to

$$
\begin{aligned}
v_{s}^{-2} \delta \mathcal{M}_{x}+(v \zeta)^{-2} \nabla^{2} \delta \mathcal{M}_{x}+\left(\delta \mathcal{B}_{x}-\delta \mathcal{M}_{x}\right)-\bar{\eta}_{1} \delta \mathcal{M}_{x}+\bar{\eta}_{2} \nabla^{2} \delta \mathcal{M}_{x}= & \left(v_{s}^{-2}-v^{-2}\right) \mathcal{M}_{0 x}+v_{s}^{-2} \mathcal{M}_{0 x}\left(\mathcal{M}_{0 x}^{2}+\mathcal{M}_{0 y}^{2}+\mathcal{M}_{0 z}^{2}\right) \\
& +2 \bar{\eta}_{1} \delta f \mathcal{M}_{0 x}-2 \bar{\eta}_{2} \nabla \cdot\left(\delta f \nabla \mathcal{M}_{0 x}\right)+v_{s}^{-2} \frac{\beta^{\prime}}{\beta_{m}} \mathcal{M}_{0 x}^{3}
\end{aligned}
$$

(The $x$ component is given here; the $y$ and $z$ components follow by cyclic permutation.) For case 1 the last term occurs only for the $z$ equation. For case 3 the $z$ equation is discarded and $\mathcal{M}_{0 z}^{2}$ is set to zero in the other two equations. Multiplying Eq. (13) by $\mathcal{M}_{0 x}$, averaging it over space, and using

$$
v_{s}^{-2}-v^{-2}=v_{0}^{2}\left(\frac{T-T_{s}}{T_{m 0}}\right),
$$

and \langle\rangle to denote spatial averaging, we obtain

$$
\begin{aligned}
v_{0}^{-2}\left(\frac{T_{s}-T}{T_{m 0}}\right)\left\langle\mathcal{M}_{0 x}^{2}\right\rangle \\
=v_{s}^{-2}\left\langle\mathcal{M}_{0 x}^{2} \mathcal{M}_{0}^{2}\right\rangle-2\left\langle\delta f\left(\mathbf{Q}_{0}^{2}+\bar{\eta}_{1} \mathcal{M}_{0}^{2}+\bar{\eta}_{2} \mid \nabla \mathcal{M}_{0} !^{2}\right)\right\rangle \\
\quad+v_{s}^{-2} \frac{\beta^{\prime}}{\beta_{m}}\left\langle\mathcal{M}_{0 x}^{4}\right\rangle .
\end{aligned}
$$

By adding similar equations for the $y$ and $z$ components and introducing structural constants $\beta_{1}, \beta_{2}$, and $\beta_{\text {aniso }}$

$$
\left\langle M_{0}^{4}\right\rangle=\beta_{1}\left\langle M_{0}^{2}\right\rangle^{2},
$$

$$
\left\langle\delta f\left(\mathbf{Q}_{0}^{2}+\bar{\eta}_{1} \boldsymbol{M}_{0}^{2}+\bar{\eta}_{2}\left|\nabla \boldsymbol{M}_{0}\right|^{2}\right)\right\rangle=\mu_{s}^{2} \beta_{2}\left\langle\boldsymbol{M}_{0}^{2}\right\rangle^{2},
$$

where $\delta f$ is the same as defined in Ref. 10, and

$$
\begin{aligned}
& \left\langle M_{0 z}^{4}\right\rangle=\beta_{\text {aniso }}^{\text {(case }{ }^{1}}\left\langle\mathcal{M}_{0}^{2}\right\rangle^{2}, \\
& \left\langle\mathcal{M}_{0 x}^{4}+\mathcal{M}_{0 y}^{4}+\mathcal{M}_{0 z}^{4}\right\rangle=\beta_{\text {aniso }}^{\text {(c) }}\left\langle\mathcal{M}_{0}^{2}\right\rangle^{2}, \\
& \left\langle\mathcal{M}_{0 x}^{4}+\mathcal{M}_{0 y}^{4}\right\rangle=\beta_{\text {aniso }}^{\text {(case }{ }^{\prime}}\left\langle\mathcal{M}_{0}^{2}\right\rangle^{2},
\end{aligned}
$$

we finally obtain

$$
\left\langle\mathcal{M}_{0}^{2}\right\rangle=\frac{\left(T_{s}-T\right) /\left(T_{m 0}-T_{s}\right)}{\beta_{1}+\left(\beta^{\prime} / \beta_{m}\right) \beta_{\text {aniso }}-(\mu v)^{2} \beta_{2}},
$$

where $\beta_{\text {aniso }} \equiv\left\{\beta_{\text {aniso }}^{\text {(case 1) }}, \beta_{\text {aniso }}^{\text {(case })}\right.$, or $\left.\beta_{\text {aniso }}^{\text {(case } 3)}\right\}$. In the above, $\beta_{1}$ and $\beta_{2}$ are of course the same as Eqs. (23a) and (23b) of Ref. 1, so only $\beta_{\text {aniso }}$ is new. Eq. (19) should be compared with Eq. (24) of Ref. 1. We will, however, neglect the $(\mu v)^{2} \beta_{2}$ term in the following analysis because this term is most likely very small for actual materials. If the $\eta_{1}$ and $\eta_{2}$ terms do not dominate in the definition for $\beta_{2}$ then $\beta_{2} \approx q_{c}^{-4} \leq 10^{-4}$. ${ }^{10}$ We have also estimated that $(\mu \nu)^{2}<100 .{ }^{14}$ Since the product $(\mu \nu)^{2} \beta_{2}$ is only marginally finite positive it will not change the 
qualitative behavior of the model and only affect the quantitative results roughly at the one percent level. This changes the denominator of Eq. (25) in Ref. 1 to give

$$
\frac{\mathcal{F}-\mathscr{F}_{s 0}}{\left|\mathfrak{F}_{s 0}\right|} \propto-\frac{1}{\mathcal{D}_{F}}
$$

where $\mathscr{D}_{F} \equiv \beta_{1}+\left(\beta^{\prime} / \beta_{m}\right) \beta_{\text {aniso. }}$. It is therefore clear that minimizing the free-energy density is completely equivalent to minimizing $\mathscr{D}_{F}$.

\section{THE EXPRESSION FOR $\mathscr{D}_{F}$}

We can resolve $\boldsymbol{M}_{0}$ to have components along the $x, y$, and $z$ axes. Since we are limiting ourselves to only singly periodic structures, we can set

$$
\mathcal{M}_{0}=\mathcal{M}_{0 q} e^{i \mathbf{q} \cdot \mathbf{r}}+\mathcal{M}_{0 q}^{*} e^{-i \mathbf{q} \cdot \mathbf{r}}
$$

where

$$
M_{0 q}=\hat{\mathbf{x}} M_{0 q, x}+\hat{\mathbf{y}} M_{0 q, y}+\hat{\mathbf{z}} M_{0 q, z} .
$$

If we define $q$ to point in a direction $\left(\theta_{q}, \phi_{q}\right)$ we can define basis vectors $\widehat{\mathbf{e}}_{1}$ and $\hat{\mathbf{e}}_{2}$ as $\hat{\mathbf{e}}_{1}=-\hat{\mathbf{x}} \sin \phi_{q}+\hat{\mathbf{y}} \cos \phi_{q}$ and $\hat{\mathbf{e}}_{2}=-\hat{\mathbf{x}} \cos \phi_{q} \cos \theta_{q}-\hat{\mathbf{y}} \sin \phi_{q} \cos \theta_{q}+\hat{\mathbf{z}} \sin \theta_{q}$, so that $\widehat{\mathbf{e}}_{1}, \hat{\mathbf{e}}_{2}$, and $\hat{\mathbf{q}}$ form a right-handed coordinate system. Since $\mathcal{M}_{0 q}$ is transverse we can always resolve it as

$$
\boldsymbol{M}_{0 q}=\hat{\mathbf{e}}_{1} M_{1}+\hat{\mathbf{e}}_{2} M_{2} \text {. }
$$

In this notation we can write

$$
\beta_{1}=\frac{4\left(\left|M_{1}\right|^{2}+\left|M_{2}\right|^{2}\right)^{2}+2\left(M_{1}^{2}+M_{2}^{2}\right)\left(M_{1}^{* 2}+M_{2}^{* 2}\right)}{4\left(\left|\mathcal{M}_{1}\right|^{2}+\left|\mathcal{M}_{2}\right|^{2}\right)^{2}} \text {, }
$$

and

$$
\beta_{\text {aniso }}^{\text {(case } 2)}=6 \frac{\left(A\left|M_{1}\right|^{4}+B\left|M_{2}\right|^{4}+C\left|M_{1}\right|^{2}\left|M_{2}\right|^{2}+D \chi\left|M_{1}\right|^{2}+E \chi\left|M_{2}\right|^{2}+F \chi^{2}\right)}{4\left(\left|M_{1}\right|^{2}+\left|M_{2}\right|^{2}\right)^{2}}
$$

where $A, B, C, D, E$, and $F$ are complicated functions of $\theta_{q}$ and $\phi_{q}$ and

$$
\chi \equiv M_{1} M_{2}^{*}+M_{1}^{*} M_{2}
$$

We can now write $D_{F}$ in terms of the magnetization basis amplitudes $M_{1}$ and $M_{2}$ :

$$
\begin{aligned}
\mathscr{D}_{F}=[ & 4\left(\left|\mathcal{M}_{1}\right|^{2}+\left|\mathcal{M}_{2}\right|^{2}\right)^{2}+2\left(\mathcal{M}_{1}^{2}+\mathcal{M}_{2}^{2}\right)\left(\mathcal{M}_{1}^{* 2}+\mathcal{M}_{2}^{* 2}\right) \\
& +6 \frac{\beta^{\prime}}{\beta_{m}}\left(A\left|\mathcal{M}_{1}\right|^{4}+B\left|\mathcal{M}_{2}\right|^{4}+C\left|\mathcal{M}_{1}\right|^{2}\left|\mathcal{M}_{2}\right|^{2}\right. \\
& \left.\left.\quad+D \chi\left|\mathcal{M}_{1}\right|^{2}+E \chi\left|\mathcal{M}_{2}\right|^{2}+F \chi^{2}\right)\right] / 4\left(\left|\mathcal{M}_{1}\right|^{2}+\left|\mathcal{M}_{2}\right|^{2}\right)^{2} \text { (case } 2 \text { only). }
\end{aligned}
$$

For a quartic anisotropy energy with cubic symmetry, $\mathscr{D}_{F}$ was minimized numerically. However, the case of uniaxial anisotropy is soluble analytically and we present it here first to give the reader a feeling of how this problem is attacked mathematically.

\section{QUARTIC ANISOTROPY WITH UNIAXIAL SYMMETRY (CASE 1)}

To study a uniaxial anisotropy, we can assume $q=\hat{\mathbf{x}} \sin \theta_{q}+\hat{\mathbf{z}} \cos \theta_{q}$ without loss of generality, and $\hat{\mathbf{e}}_{1}=\hat{\mathbf{y}}$, $\widehat{\mathbf{e}}_{2}=-\widehat{\mathbf{x}} \cos \theta_{q}+\hat{\mathbf{z}} \sin \theta_{q}$, with $\hat{\mathbf{z}}$ being along the anisotropy axis. Then the reduced anisotropy energy after spacial averaging is simply

so

$$
6 \frac{\beta^{\prime}}{\beta_{m}}\left|M_{0 q, z}\right|^{4}=6 \frac{\beta^{\prime}}{\beta_{m}}\left|M_{2} \sin \theta_{q}\right|^{4}
$$

$$
D_{F}=\frac{4\left(\left|\mathcal{M}_{1}\right|^{2}+\left|\mathcal{M}_{2}\right|^{2}\right)^{2}+2\left(\mathcal{M}_{1}^{2}+\mathcal{M}_{2}^{2}\right)\left(\mathcal{M}_{1}^{* 2}+\mathcal{M}_{2}^{* 2}\right)+6 \frac{\beta^{\prime}}{\beta_{m}}\left|\mathcal{M}_{2}\right|^{4} \sin ^{4} \theta_{q}}{4\left(\left|\mathcal{M}_{1}\right|^{2}+\left|\mathcal{M}_{2}\right|^{2}\right)^{2}} .
$$

Let $\mathcal{M}_{1}=\mathcal{M}_{0} \cos \alpha e^{i \gamma_{1}}$, and $\mathcal{M}_{2}=\mathcal{M}_{0} \sin \alpha e^{i \gamma_{2}}$. This leads to

$$
\mathcal{D}_{F}=\frac{3}{2}-\frac{1}{2} \sin ^{2}(2 \alpha) \sin ^{2}\left(\gamma_{1}-\gamma_{2}\right)+\frac{A^{\prime}}{2} \sin ^{4} \alpha
$$

where $A^{\prime} \equiv 3\left(\beta^{\prime} / \beta_{m}\right) \sin ^{4} \theta_{q}$. Minimization of $D_{F}$ with respect to $\left(\gamma_{1}-\gamma_{2}\right)$ clearly leads to $\left(\gamma_{1}-\gamma_{2}\right)=\pi / 2$, $\sin ^{2}\left(\gamma_{1}-\gamma_{2}\right)=1$. A further minimization with respect to $\sin ^{2} \alpha$ then leads to

$$
\sin ^{2} \alpha_{\text {opt }}=\frac{2}{A^{\prime}+4}
$$

which is meaningful only for $A^{\prime} \geq-2$. If $A^{\prime}<-2$, we must take $\sin ^{2} \alpha_{\text {opt }}=1$. This gives 


$$
\min \mathcal{D}_{F}=\frac{3}{2}-\frac{2}{A^{\prime}+4} \quad\left(\text { for } A^{\prime} \geq-2\right),
$$

and

$$
\min \mathcal{D}_{F}=\frac{3}{2}+\frac{A^{\prime}}{2} \quad\left(\text { for } A^{\prime} \leq-2\right) .
$$

Equations (32) and (33) together represent a single monotonically increasing function of $A^{\prime}$. This allows us to divide the physical region of $\beta^{\prime} / \beta_{m}$, viz., $\beta^{\prime} / \beta_{m}>-1$, into three regions.

Region I. $-1<\beta^{\prime} / \beta_{m} \leq-\frac{2}{3}$. Since $3 \beta^{\prime} / \beta_{m} \leq A^{\prime} \leq 0$ here, the minimum $\mathcal{D}_{F}$ with respect to $\theta_{q}$ is at $A^{\prime}=3 \beta^{\prime} / \beta_{m} \leq-2$ for $\theta_{q}=90^{\circ}$, giving $\sin ^{2} \alpha=1$ with $D_{F}=\frac{3}{2}\left[1+\left(\beta^{\prime} / \beta_{m}\right)\right]$. The solution is LP with $\mathcal{M}$ parallel to $\widehat{\mathbf{z}}$, and $\mathbf{q}$ in any direction perpendicular to $\widehat{\mathbf{z}}$.

Region II. $-\frac{2}{3}<\beta^{\prime} / \beta_{m}<0$. Here again $3 \beta^{\prime} / \beta_{m}$ $\leq A^{\prime} \leq 0$, so the minimum $D_{F}$ is at $A^{\prime}=3 \beta^{\prime} / \beta_{m}>-2$, which gives $\theta_{q}=90^{\circ}$ and $\sin ^{2} \alpha_{(\mathrm{opt})}=2 /\left[3\left(\beta^{\prime} / \beta_{m}\right)+4\right]<1$ corresponding to EP. In fact, as $\beta^{\prime} / \beta_{m}$ goes from 0 to $-\frac{2}{3}$, the polarization changes continuously from $\mathrm{CP}$ to LP. On this interval $\mathscr{D}_{F}=\left[8+9\left(\beta^{\prime} / \beta_{m}\right)\right] /$ $\left[8+6\left(\beta^{\prime} / \beta_{m}\right)\right]$. In this state, $M$ spirals in a plane parallel to $\hat{\mathbf{z}}$, and $\hat{\mathbf{q}}$ is perpendicular to this plane.

Region III. $\beta^{\prime} / \beta_{m}>0$. In this region $0 \leq A^{\prime}$ $\leq 3 \beta^{\prime} / \beta_{m}$, the minimum of $D_{F}$ is at $A^{\prime}=0$ which implies $\theta_{q}=0$. As a result $\sin ^{2} \alpha_{(\text {opt })}=\frac{1}{2}$ implying CP and $D_{F}=1$. Here, $\boldsymbol{M}$ spirals in the plane perpendicular to $\hat{\mathbf{z}}$, and $\hat{\mathbf{q}}$ is therefore along $\hat{\mathbf{z}}$. Combining the three regions gives the behavior of the free-energy denominator for the case of uniaxial anisotropy energy as given in Fig. 1. Note that for this case the polarization phase transition in the negative $\beta^{\prime} / \beta_{m}$ region is such that $\mathscr{D}_{F}$ and its first derivative are continuous functions of $\beta^{\prime} / \beta_{m}$.

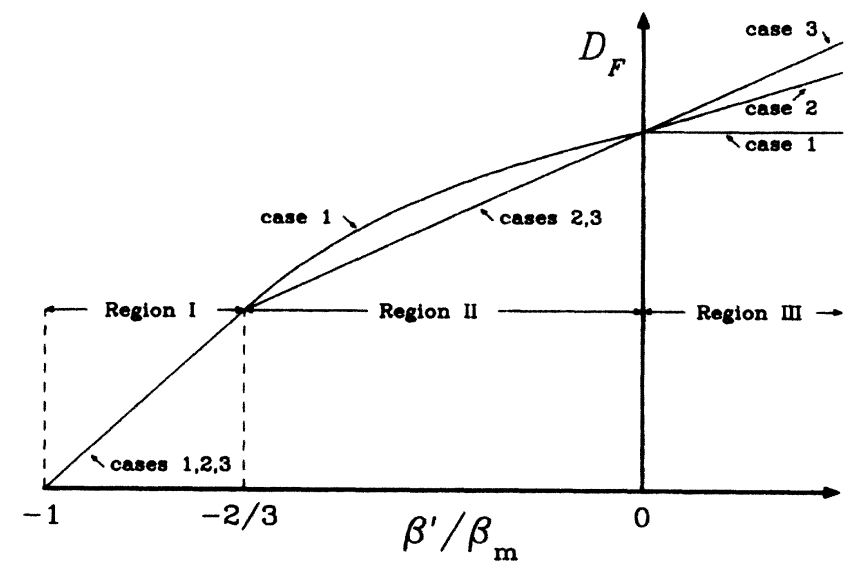

FIG. 1. Plot of free-energy denominator vs anisotropy strength for three types of quartic anisotropy energy.

\section{QUARTIC ANISOTROPY WITH CUBIC SYMMETRY (CASE 2)}

Now we return to the full cubic anisotropy, Eq. (1b). The reduced anisotropy energy after spatial averaging is

$$
6 \frac{\beta^{\prime}}{\beta_{m}}\left(\left|M_{0 q, x}\right|^{4}+\left|M_{0 q, y}\right|^{4}+\left|M_{0 q, z}\right|^{4}\right) .
$$

Of course we will actually use the decomposition Eq. (23) to solve this case. As in the previous case we set

$$
\begin{aligned}
& M_{1}=M_{0}(\cos \beta) e^{i \gamma_{1}}, \\
& M_{2}=M_{0}(\sin \beta) e^{i \gamma_{2}} .
\end{aligned}
$$

Then Eq. (27) simplifies to

$$
\begin{aligned}
\mathscr{D}_{F}=\frac{3}{2}-\frac{1}{2} \sin ^{2}(2 \beta) \sin ^{2}\left(\gamma_{1}-\gamma_{2}\right)+\frac{3}{2}\left(\beta^{\prime} / \beta_{m}\right)[ & A \cos ^{4} \beta+B \sin ^{4} \beta+C \cos ^{2} \beta \sin ^{2} \beta \\
& +2 D \cos \left(\gamma_{1}-\gamma_{2}\right) \cos ^{3} \beta \sin \beta+2 E \cos \left(\gamma_{1}-\gamma_{2}\right) \sin ^{3} \beta \cos \beta \\
& \left.+4 F \cos ^{2}\left(\gamma_{1}-\gamma_{2}\right) \cos ^{2} \beta \sin ^{2} \beta\right] .
\end{aligned}
$$

We can only minimize this expression numerically. (First with respect to $\gamma_{1}-\gamma_{2}$ and $\beta$, and then with respect to $\theta_{q}$ and $\phi_{q}$.) We find a phase transition in polarization at $\beta^{\prime} / \beta_{m}=-\frac{2}{3}$. The polarization is $C P$ for $\beta^{\prime} / \beta_{m}$ between 0 and $-\frac{2}{3}$, but it suddenly chooses LP at $-\frac{2}{3}$, and remains so down to the physical limit of $\beta^{\prime} / \beta_{m}=-1$. The physical region $\beta^{\prime} / \beta_{m}>-1$ can therefore again be divided into three regions as given below.

Region I. $-1<\beta^{\prime} / \beta_{m} \leq-\frac{2}{3}$. We find $\mathbf{q}$ favoring whole $x y, y z$, and $z x$ planes. When $\mathrm{q}$ favors the cubic $y z$ and $z x$ planes, $\beta=0$ which implies that the magnetization is all $M_{1}$ from Eq. (35). When $\mathrm{q}$ favors the cubic $x y$ plane, $\beta=\pi / 2$ which implies that the magnetization is all $M_{2}$ from Eq. (36). In both situations, $M$ turns out to be along a cubic axis and $q$ is in the plane perpendicular to $M$ but otherwise undetermined. In this region the minimum value is $D_{F}=\frac{3}{2}\left(1+\beta^{\prime} / \beta_{m}\right)$.

Region $I I$. $-\frac{2}{3}<\beta^{\prime} / \beta_{m}<0$. We find minimum $D_{F}$ 's for $q$ along any of the principal axes, $\boldsymbol{M}$ is $C P$ in the principal plane $\perp \mathbf{q}$, and the minimum value is $\mathcal{D}_{F}$ $=1+\frac{3}{4} \beta^{\prime} / \beta_{m}$.

Region III. $\beta^{\prime} / \beta_{m}>0$. We find minimum $\mathscr{D}_{F}$ 's at $q=(111)$ and its equivalent in the other octants. The qualitative behavior of the solution remains the same for all positive values of $\beta^{\prime} / \beta_{m}$, and is $C P$ with $\boldsymbol{M}$ in the plane perpendicular to $q$. Here the minimum is $D_{F}=1+\frac{1}{2} \beta^{\prime} / \beta_{m}$. Figure 1 shows the behavior of $\mathscr{D}_{F}$ in each of the three regions. 


\section{ANISOTROPY MODEL FOR ErRh ${ }_{4} B_{4}$ (CASE 3)}

We now consider the anisotropy of $\mathrm{ErRh}_{4} \mathrm{~B}_{4}$. Here, the $z$ axis is the hard axis with a much lower $T_{m 0}^{(z)}$ so the magnetization is confined to the $x-y$ plane. The quartic anisotropy energy for this case is, after spatial averaging,

$$
6 \frac{\beta^{\prime}}{\beta_{m}}\left(\left|\mathcal{M}_{0 q, x}\right|^{4}+\left|M_{0 q, y}\right|^{4}\right) .
$$

Here no new coordinate system is needed, and we can directly work with $\mathcal{M}_{0 q, x}$ and $\mathcal{M}_{0 q, y}$. We need to minimize

$$
\begin{aligned}
\mathscr{D}_{F}= & {\left[4\left(\left|\mathcal{M}_{0 q, x}\right|^{2}+\left|\mathcal{M}_{0 q, y}\right|^{2}\right]^{2}+2\left(\mathcal{M}_{0 q, x}^{2}+\mathcal{M}_{0 q, y}^{2}\right)\left(\mathcal{M}_{0 q, x}^{* 2}+\mathcal{M}_{0 q, y}^{* 2}\right)\right.} \\
& \left.+6 \frac{\beta^{\prime}}{\beta_{m}}\left[\left|\mathcal{M}_{0 q, x}\right|^{4}+\left|\mathcal{M}_{0 q, y}\right|^{4}\right)\right] / 4\left(\left|\mathcal{M}_{0 q, x}\right|^{2}+\left|\mathcal{M}_{0 q, y}\right|^{2}\right)^{2}
\end{aligned}
$$

$$
\begin{aligned}
& \text { Now let } \mathcal{M}_{0 q, x}=\mathcal{M}_{0}(\cos \phi) e^{i \gamma_{x}} \\
& =\mathcal{M}_{0}(\sin \phi) e^{i \gamma_{y}} \text {. This leads to } \\
& \mathscr{D}_{F}=1+\left[\frac{1}{2}+\frac{3 \beta^{\prime}}{2 \beta_{m}}\right]\left(\cos ^{4} \phi+\sin ^{4} \phi\right) \\
& +\cos 2\left(\gamma_{x}-\gamma_{y}\right) \cos ^{2} \phi \sin ^{2} \phi .
\end{aligned}
$$

Since $\cos ^{2} \phi \sin ^{2} \phi$ is nonnegative for all $\phi$, we can immediately minimize $\mathscr{D}_{F}$ with respect to the relative phase $\gamma_{x}-\gamma_{y}$ to give

$$
\gamma_{x}-\gamma_{y}=\frac{\pi}{2}
$$

and

$$
D_{F}=1+\left(\frac{1}{2}+\frac{3 \beta^{\prime}}{2 \beta_{m}}\right)\left(\cos ^{4} \phi+\sin ^{4} \phi\right)-\cos ^{2} \phi \sin ^{2} \phi .
$$

A further minimization of $\mathscr{D}_{F}$ with respect to $\phi$ indicates CP (i.e., $\sin ^{2} \phi=\cos ^{2} \phi=\frac{1}{2}$ ) if $\beta^{\prime} / \beta_{m}>-\frac{2}{3}$. If, however, $-1<\beta^{\prime} / \beta_{m}<-\frac{2}{3}$, the magnetization is LP (i.e., $\sin ^{2} \phi=0$ or 1 ). We can divide the physical range of $\beta^{\prime} / \beta_{m}$ into two regions.

Region I. $-1<\beta^{\prime} / \beta_{m} \leq-\frac{2}{3}$. The polarization is LP with the magnetization along either of the $x$ and $y$ axes and the wave vector $q$ in any direction perpendicular to the magnetization. On this interval $D_{F}=\frac{3}{2}\left(1+\beta^{\prime} / \beta_{m}\right)$.

Region II. $\beta^{\prime} / \beta_{m}>-2 / 3$. The polarization is CP with $\mathrm{q}$ in the $z$ direction. On this interval, $D_{F}=1$ $+3 \beta^{\prime} / 4 \beta_{m}$. The behavior of $\mathcal{D}_{F}$ for the two regions is shown in Fig. 1. Note that there is a first order polarization phase transition at $\beta^{\prime} / \beta_{m}=-\frac{2}{3}$.

\section{CONCLUSIONS}

We have analyzed the effect of adding three types of quartic anisotropy energy (called cases 1, 2, and 3) on the polarization of the spiral magnetic coexistence state of superconductivity and ferromagnetism. This state was originally proposed by Blount and Varma based on an isotropic Ginzburg-Landau-type theory, and previously only the effect of a quadradic anisotropy energy on this state has been analyzed.

In case 1 , the quartic anisotropy has a uniaxial sym- metry. We find only circular polarization for the unihard-axis subcase, but for the uni-easy-axis subcase, continuous polarization transitions from circular to elliptic to linear are found as the strength of anisotropy is increased. The transition from elliptical polarization to linear is a genuine second-order transition, whereas the circular polarization is merely a limiting case of the elliptic polarization as the strength of anisotropy goes to zero, which does not correspond to a genuine phase transition. For this case and the next two cases, we have also determined the optimum directions of magnetization $\mathbf{M}$ and wave vector $q$ at each value of the anisotropy strength.

In case 2 , the quartic anisotropy has a cubic symmetry. Again, only circular polarization is obtained if the sign of the anisotropy energy is to work against the cubic axes, but when the cubic axes are favored, we find a direct polarization transition from circular to linear without going through an intermediate elliptic stage. This transition is a genuine first-order transition.

Finally, case 3 is to emulate the actual anisotropy of primitive tetragonal $\mathrm{ErRh}_{\mathbf{4}} \mathrm{B}_{\mathbf{4}}$. A quartic anisotropy of square symmetry in the tetragonal basal $(x y)$ plane is combined with the assumption that the bare Curie temperature is much lower for magnetization in the hard tetragonal $c$ or $z$ axis. Since this study is confined to the vicinity of the onset temperature of the coexistence state, this case reduces simply to the two-dimensional analog of the previous case. The conclusion is also found to be very similar, in that a discontinuous circular to linear polarization transition is found at a finite strength of the anisotropy energy within its stability limit, if the sign of the anisotropy is to favor the tetragonal $a$ axes.

In cases 1 and 2 , but not in case 3 , a more obvious transition is also found as the anisotropy energy changes its sign, where the polarization does not change (i.e. remains circular on both sides), but the optimum directions of $\mathbf{M}$ and $\mathbf{q}$ change discontinuously. The behavior of all three cases is summarized in Table $I$.

One behavior of our results can be easily understood, which brings contact with an earlier work. For $\beta^{\prime} / \beta_{m}<-\frac{2}{3}$ for all three cases we find that $\mathscr{D}_{F}$ $=\frac{3}{2}\left[\left(\beta_{m}+\beta^{\prime} / \beta_{m}\right]\right.$. This gives exactly the free-energy result of Ref. 10 for the linearly polarized plane-wave-like structure but with $\beta_{m}$ there replaced by $\beta_{m}+\beta^{\prime}$ here, as it should be, since the $\beta_{m}$ there is the effective coefficient of $\frac{1}{4} M_{z}^{4}$. 
TABLE I. Summary of the effect of three types of quartic anisotropy energy on the "spiral magnetic" coexistence state of superconductivity and ferromagnetism, where CP, EP, and LP denote circular, elliptic, and linear polarizations, respectively.

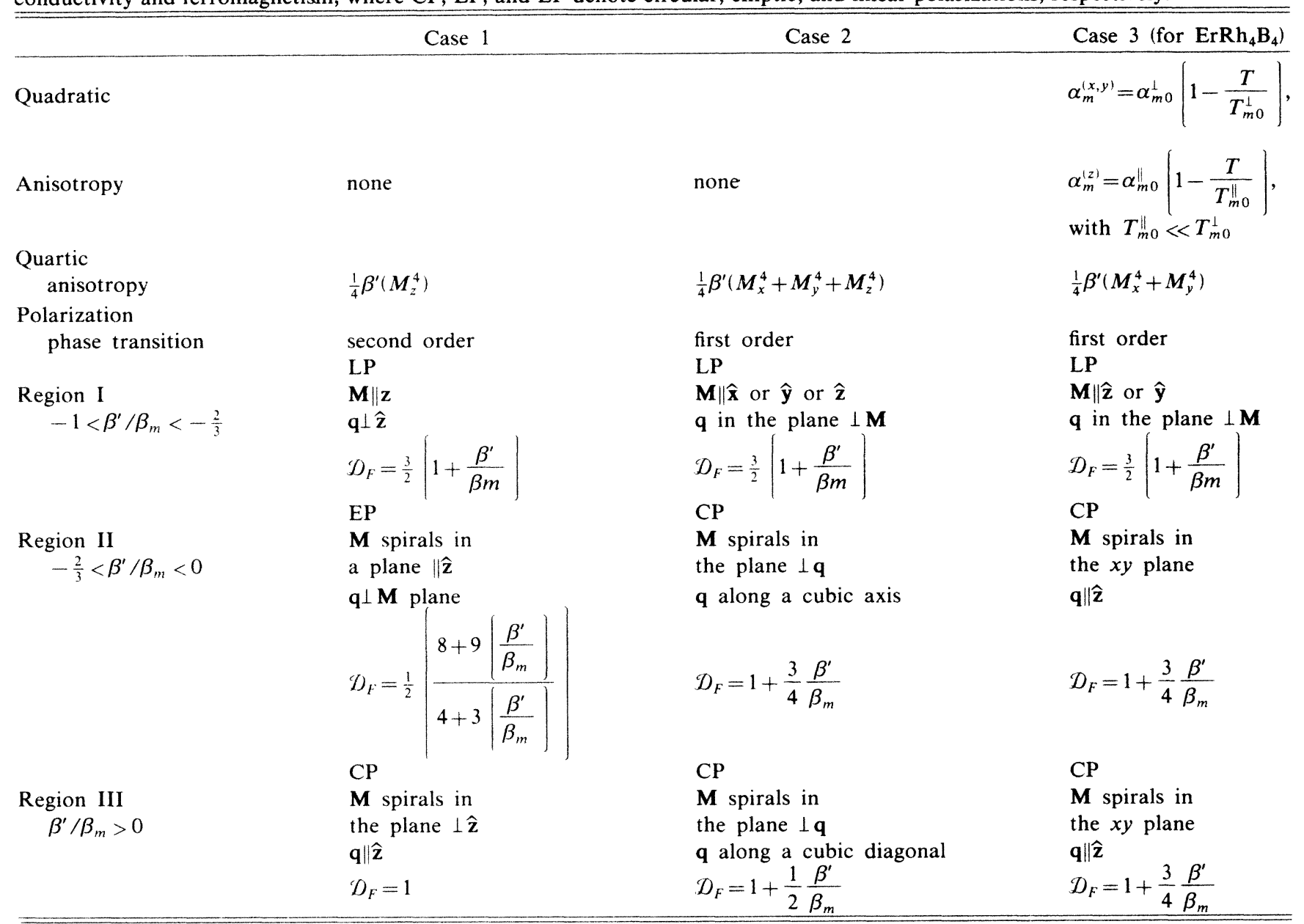

It is interesting to note that the present theory predicts that a circularly polarized spiral magnetic state can persist for a finite range of the quartic anisotropy energy strength, when it has three equivalent mutually orthogonal easy axes, since for the single-easy-axis case the state will turn elliptically polarized at an infinitesimal anisotropy strength. Another interesting prediction of this work is that the polarization remains circular for any strength of the quartic anisotropy as long as it has a cubic symmetry and favors eight easy directions along the cubic diagonals. Finally, and most importantly, we have presented in this work a simple anisotropy model which can explain why linear polarization may occur in the coexistence state of primitive tetragonal $\mathrm{ErRh}_{4} \mathrm{~B}_{4}$. The present work also shows that ferromagnetic superconductors with a cubic symmetry have some special interest, but unfortunately, only antiferromagnetic superconductors with a cubic symmetry have been found so far. ${ }^{15}$

\section{ACKNOWLEDGMENTS}

This work was partially supported by the National Science Foundation Grant No. DMR82-05697. We would like to thank J. W. Lynn for useful discussions on the anisotropy properties of the Chevral phase compounds. One of us (G.H.R.) is a recipient of a Z. M. and W. Wilson fellowship.
${ }^{1}$ E. I. Blount and C. M. Varma, Phys. Rev. Lett. 42, 1079 (1979).

${ }^{2}$ D. E. Moncton, D. B. McWhan, P. H. Schmidt, G. Shirane, W. Thomlinson, M. B. Maple, H. B. MacKay, L. D. Woolf, Z. Fisk, and D. C. Johnson, Phys. Rev. Lett. 45, 2060 (1980).

${ }^{3}$ J. W. Lynn, G. Shirane, W. Thomlinson, and R. N. Shelton, Phys. Rev. Lett. 46, 368 (1981).

${ }^{4}$ G. W. Crabtree, F. Behroozi, S. A. Cambell, and D. G. Hinks,
Phys. Rev. Lett. 49, 1342 (1982).

${ }^{5}$ S. K. Sinha, G. W. Crabtree, D. G. Hinks, and H. Mook, Phys. Rev. Lett. 48, 950 (1982).

${ }^{6} \emptyset$. Fisher, Appl. Phys. 16, 1 (1978).

${ }^{7}$ J. W. Lynn, D. E. Moncton, W. Thomlinson, G. Shirane, and R. N. Shelton, Solid State Commun. 26, 493 (1978); F. Holtzberg, S. J. LaPlaca, T. R. McGuire, and R. A. Webb, J. Appl. Phys. 55, 2013 (1984); F. Holtzberg, R. A. Webb, T. 
R. McGuire, and S. J. LaPlaca, ibid. 57, 3121 (1985); and J. Rossat-Mignod, P. Burlet, S. Quezel, A. Benoit, J. Flouquet, R. Horyn, O. Pena, and M. Sergent, J. Phys. Lett. (Paris) (to be published).

${ }^{8}$ H. S. Greenside, E. I. Blount, and C. M. Varma, Phys. Rev. Lett. 46, 49 (1981); and H. S. Greenside, Ph. D. dissertation, Princeton University, 1981.

${ }^{9}$ C. G. Kuper, M. Revzen, and A. Ron, Solid State Commun. 36, 533 (1980).

${ }^{10}$ C.-R. Hu, Phys. Rev. B 30, 2582 (1984).

11 J. W. Lynn (private communication).

12 J. W. Lynn, J. A. Gotaas, R. W. Erwin, R. A. Ferrell, J. K. Bhattacharjee, R. N. Shelton, and P. Klavins, Phys. Rev. Lett. 52, 133 (1984).

${ }^{13}$ J. M. Vandenberg and B. T. Matthias, Proc. Natl. Acad. Sci. USA 74, 1336 (1977).
${ }^{14} \mathrm{We}$ estimate $(\mu \nu)^{2}$ as follows: Since it is a weakly temperature-dependent factor [c.f. Ref. 10, Eqs. (7a) and (7b)], it can be estimated as a product of two factors at $T=0$. The factor $(\mu / v)^{2}$ at $T=0$ is just the factor $\zeta$ of C. G. Kuper, M. Revzen, A. Ron, and C. R. Hu, Phys. Rev. B 29, 466 (1984), and is of the order 3-17 [cf. Ref. 10, Eq. (3)]. The factor $v^{4}$ at $T=0$ is equal to $\left(4 \pi /\left|\alpha_{m 0}\right|\right)^{2}$ $\approx(4 \pi / 5.5)^{2}=5.22$ [cf. F. Behroozi, G. W. Crabtree, S. A. Campbell, D. R. Snider, S. Sihneider, and M. Levy, J. Low Temp. Phys. 49, 73 (1980)]. Together we obtain the estimate of $(\mu v)^{2}$ to be $\sim 90$ or less.

${ }^{15}$ See, for example, H. A. Kierstead, B. D. Dunlap, S. K. Malik, A. M. Umarji, and G. K. Shenoy, Phys. Rev. B 32, 135 (1985); R. N. Shelton, L. S. Hausermann-Berg, M. J. Johnson, B. Klavins, and H. D. Yang, ibid. 34, 199 (1986). 